\title{
Efficient Feature Tracking of Time-varying Surfaces Using Multi-scale Motion Flow Propagation
}

\author{
Bin Liao ${ }^{\mathrm{a}, \mathrm{b}}$, Chunxia Xiao ${ }^{\mathrm{a}, *}$, Zhiqiang Pang $^{\mathrm{a}}$ \\ ${ }^{a}$ School of Computer, Wuhan University, Wuhan 430072, China \\ ${ }^{b}$ Faculty of Mathematics and Computer Science, Hubei University, Wuhan 430062, China
}

\begin{abstract}
This paper presents a framework for efficient feature tracking of time-varying surfaces. The framework can not only capture the dynamic geometry features on the time-varying surfaces, but also can compute the accurate boundaries of the geometry features. The basic idea of the proposed approach is using the multi-scale motion flow and surface matching information to propagate the feature frame on the time-varying surfaces. We first define an effective multi-scale geometry motion flow for the time-varying surfaces, which efficiently propagates the geometry features along the time direction of the time-varying surfaces. By combining both the approximately invariant signature vectors and geometry motion flow vectors, we also incorporate the shape matching into the system to process feature tracking for time-varying surfaces in large deformation while with low frame sampling rate. Our approach does not depend on the topological connection of the underlying surfaces. Thus,it can process both mesh-based and point-based time-varying surfaces without vertex-to-vertex correspondence across the frames. Feature tracking results on different kinds of time-varying surfaces illustrate the efficiency and effectiveness of the proposed method.
\end{abstract}

Keywords: Multi-scale filtering, time-varying surfaces, geometry feature tracking, geometry motion flow, Gaussian mixture model

\footnotetext{
*Corresponding author

Email address: cxxiao@whu.edu.cn (Chunxia Xiao)
} 


\section{Introduction}

Time-varying surfaces, also called animated objects or dynamic shapes, are given as a sequence of surface frames, each of which is a static geometry model (mesh based surface or point based surface). Recently, with the progress of advanced scanning technologies, it is possible to reconstruct timevarying surfaces from the point clouds scanned for the deformed or animated objects [1]. Time-varying surfaces are more expressive compared with static geometry model, and they are now a popular data representation method with wide applications in various fields, such as virtual reality, computer games, 3D animated films, product design and scientific computation visualization. As a promising data representation, a large number of research work on time-varying surfaces have been proposed, such as segmentation $[2,3,4]$, geometry reconstruction $[1,5,6]$, geometry editing and modeling $[7,8]$.

In this paper, we aim at tracking geometry features of time-varying surfaces, namely, tracking the features' motion paths of time-varying surfaces. Similar to video object tracking [9], efficient feature tracking of time-varying surfaces is one of the critical tasks in many geometry processing applications such as geometry editing and modeling, geometry transmission, feature detection, and geometry description. However, unlike the video tracking, where there are pixel-to-pixel correspondences among the consequent image frames, time-varying surfaces such as point-based geometry usually do not hold the point-to-point correspondence among adjacent surfaces. Feature tracking of time-varying surfaces is a challenging job considering the following aspects:

- Capturing the dynamic geometry features with accurate boundaries over time-varying surfaces is difficult, especially when the boundaries of time-varying feature are fuzzy.

- Time-varying surfaces usually do not hold the point-to-point correspondence among adjacent surfaces, especially for point sampled geometry sequences, which increases the complexity of features tracking of timevarying surfaces.

- Data amount of time-varying surface is usually much larger than the single static object, thus the efficiency of feature tracking is one performance bottleneck which needs to be addressed.

To address aforementioned problems, we present a novel framework for efficient feature tracking of time-varying surfaces. The basic idea of the 
presented approach is to compute an efficient multi-scale geometry motion field for the time-varying surfaces, which propagates the current geometry feature to the next frame according to the motion field variation. For timevarying surfaces with large deformation while with low frame sampling rate, we first perform surface matching between the neighboring frames, and then combine both the surface matching results and geometry motion flow vectors for accurate feature tracking. We build localized classifiers based on GMMs (Gaussian Mixture Models) over initial boundaries of the tracked feature to compute accurate and smooth feature boundaries. Our approach does not depend on the topological connection of the underlying surfaces, thus, it can process both mesh-based and point-based time-varying surfaces.

This paper introduces the following contributions:

- Present a multi-scale geometry motion flow for efficient feature tracking of time-varying surfaces, which can process both mesh-based and pointbased time-varying surfaces without vertex-to-vertex correspondence across the frames.

- Integrate shape matching in the feature tracking system to process time-varying surfaces in large deformation with low frame sampling rate.

The rest of our paper is organized as follows. Section 2 reviews related work. Section 3 gives single-frame features and multi-frame propagation method. In section 4, we show experimental results and discussions. Finally, we conclude our paper in section 5 .

\section{Related work}

Many works on geometry processing of time-varying surfaces have been proposed, the comprehensive overview for these topics is beyond the scope of this paper. We only give the most related works such as feature tracking, shape matching, and surface reconstruction of the time-varying surfaces.

Several methods have been proposed for feature tracking of time-varying surfaces. Some approaches use strong shape prior knowledge to find features tracking of time-varying surface, such as parametric models [10, 11], machine learning [12] or modal approaches [13]. Vlasic et al.[10] and Gall et al.[11] optimized the pose of skeletal models before fitting the surface to visual data. In the case of data-driven time-varying face surfaces, Chai et 
al.[12] tracked a low number of facial features. By using machine learning techniques and a database of laser scanned faces, they mapped these features back onto a linear combination of previously acquired high resolution motion data to complete the feature tracking. Salzmann et al.[13] tracked a piece of 3D deforming cloth, and they applied Principal Component Analysis on a randomly generated set of possible configurations to constrain the space of acceptable deformations and to reduce the optimization complexity. One drawback of these approaches is that they depend on the strong shape prior knowledge.

Other approaches $[14,15]$ used little prior knowledge to perform geometry features tracking for time-varying surfaces. For example, segmentation methods [15] applied vertex positions optimization techniques and used prior knowledge as a regularization term to control vertex moving. However, these two methods operated at each vertex and would lead to difficult non-linear optimization for time-varying surfaces with strong noise. Patch based mesh tracking method [15] can receive satisfactory tracking optimization for timevarying surfaces with local deformation, however, this method assumed that the local deformations should be rigid transformations, and may not work well for tracking non-rigid features.

In fluid simulation community, many methods have been presented to track fluid surfaces. These method usually track topology-changing evolving surfaces incorporating with velocity fields or prescribed motions. Level set methods [16] and particle level set methods [17] are two preventative techniques for describing a dynamic implicit surface. These two methods consider the zero level set of a voxelized signed distance function, and integrate velocity information into the distance function to move the function, which can effectively track the surface evolution. Mesh-based surface tracking methods in surface evolvers also have been proposed, the idea behind these techniques is to evolve a triangle mesh according to a velocity field [18]. More recently, Hansen et al.[19] tracked temporally coherent, deforming triangle mesh with topology changing. They solved this problem using the surface geometry alone, without prior information like surface templates or velocity fields.

Shape matching is critical for geometry processing, a variety of methods have been proposed, refer to [20] for a survey. Shape matching between inconsistent surfaces is also critical for dynamic geometry processing and reconstruction, several methods also have been proposed $[8,21,22]$. Inspired by the SIFT method [23], Li et al.[24] extracted feature points and proposed a multi-scale representation for the surface matching, in our paper, 
we will borrow some shape matching ideas from [24] for our feature tracking. Mitra et al.[5] computed a globally consistent motion spanning multiple frames for shape matching. They added a time coordinate to all the input points based on the ordering of the respective frames, and considered the problem of computing the motion of each frame as an estimation of certain kinematic properties of the resulting space-time surfaces. Wand et al.[6] estimated a globally consistent template matching model for the reconstruction of deforming geometry from unstructured noisy point cloud sequences, they later improved their method in [25]. More recently, Li et al.[1] proposed a temporally coherent completion method for scanned dynamic shapes using a non-rigid surface registration algorithm.

There are some other works that are closely related to ours, such as tracking and modeling of non-rigid objects [26], surface reconstruction for time-varying surfaces [27, 6], and performance capture [28, 29, 30, 31, 32]. For example, Torresani et al.[26] computed the deformable template and the motion directly from the image sequences. They recovered non-rigid structure from one single video assuming the shape lies within a linear subspace. This method is promising and work well from regular video streams. Sharf et al.[27] introduced a volumetric space-time technique for the reconstruction of deforming objects from point data, they described the motion of the object as an incompressible flow of material through time, and reconstructed coherent sequences of watertight models from incomplete scanner data. Bradley et al.[28] and De Aguiar et al. [29] took advantage of temporal information to deform or to track key models for topology-preserving reconstruction.

\section{Feature tracking of time-varying surfaces}

We assume that the initial frame of the features in time-varying surfaces have been extracted, which can be specified by interactive features segmentation $[33,34]$. Then with input time-varying surfaces without vertex-to-vertex correspondence across the frames, the desired output is the tracked dynamic feature with accurate boundaries over time-varying surfaces. In order to efficiently track the dynamic features of time-varying surfaces, we first compute the multi-scale geometry motion flow vectors $\vec{v}$ for the time-varying surfaces, and with the motion flow vectors, we track the geometry features by propagating the features to the successive frames. Then we build localized classifiers based on GMMs to compute more accurate feature boundaries. Finally, we integrate surface matching method into the tracking system to track 


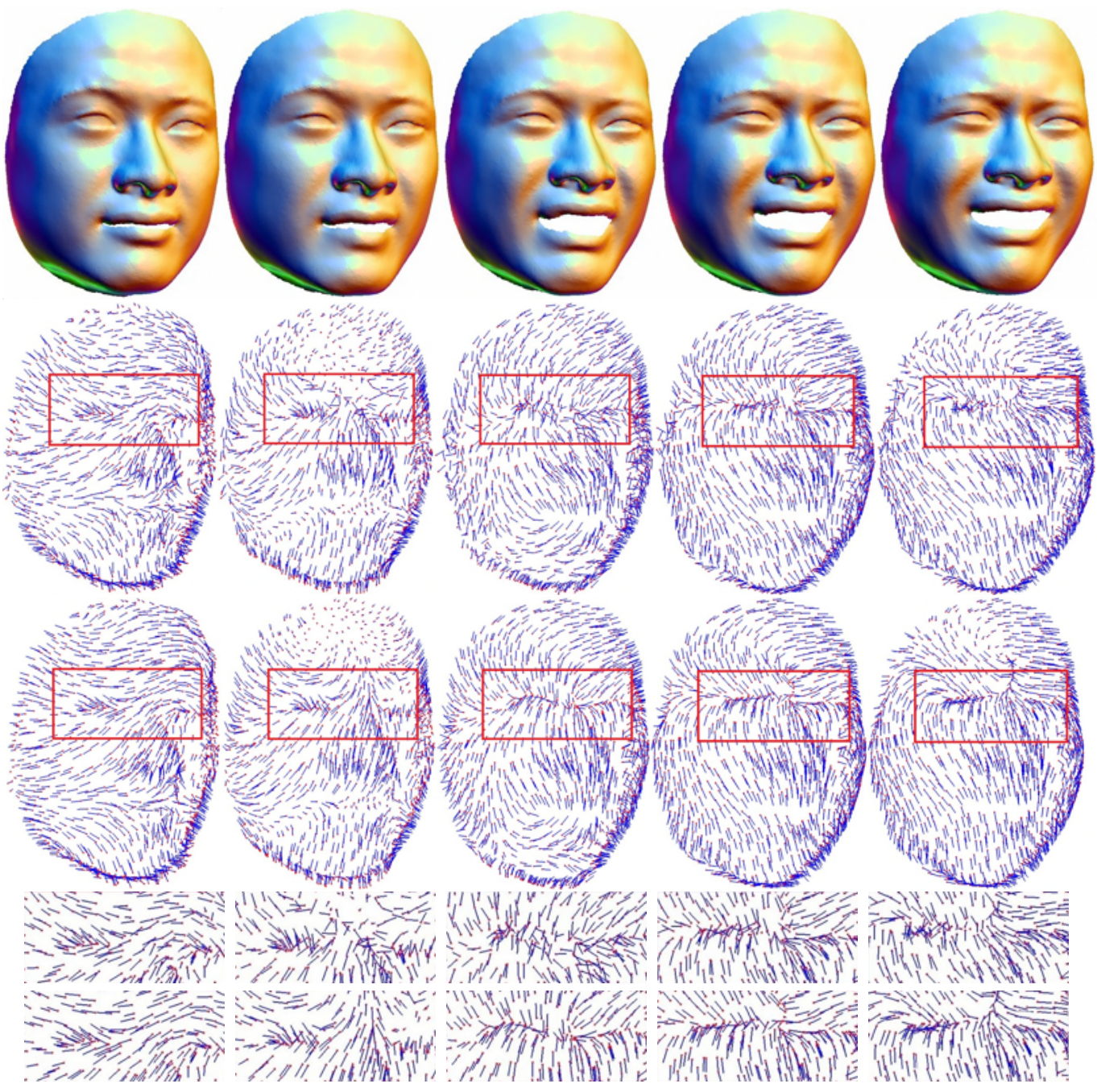

Figure 1: Velocity vectors estimation for time-varying surfaces. Top row gives 5 consequent frames in the time-varying surfaces, the second and the third row give the corresponding velocity vectors computed using single-scale strategy and multi-scale strategy, respectively. The fourth and fifth row are the zoom in results of the second and the third row, respectively. Zoom in the pictures to see the difference.

dynamic features in large deformation with low frame sampling rate. 


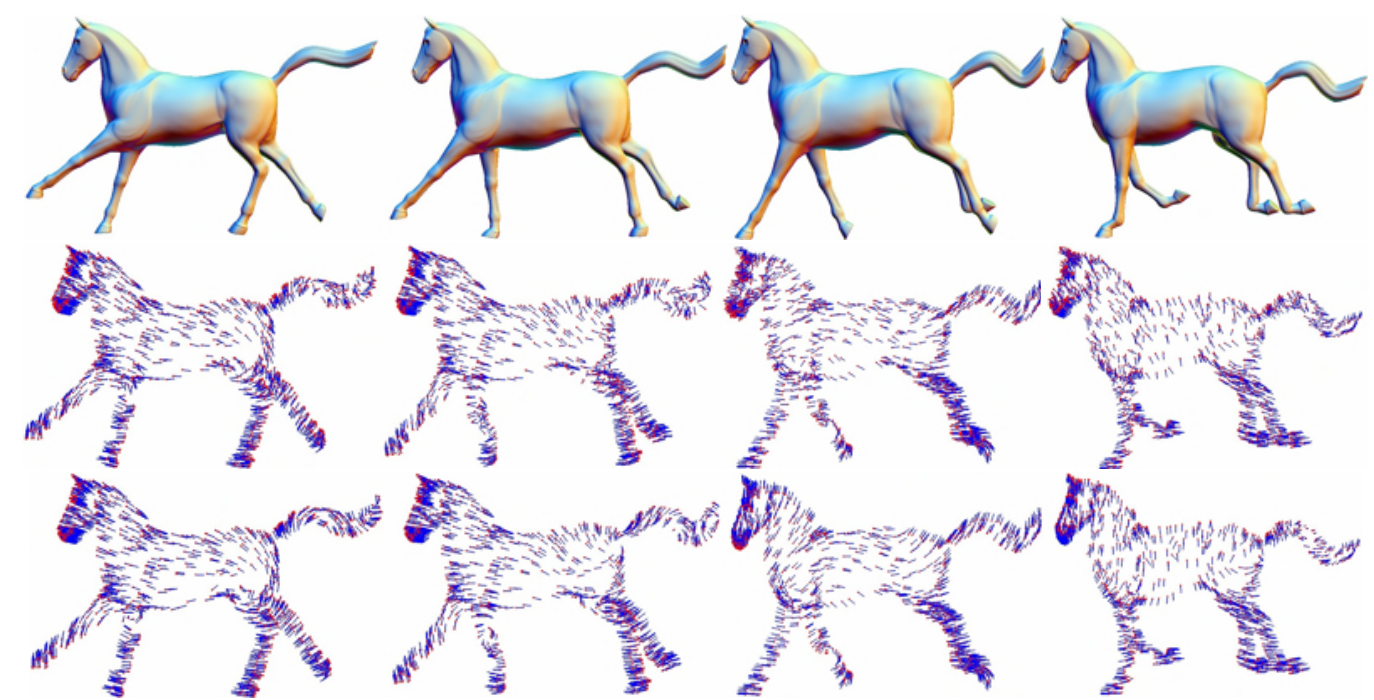

Figure 2: Velocity vectors estimation for time-varying surfaces. The middle row and the bottom row gives the corresponding velocity vectors computed using single-scale strategy and multi-scale strategy, respectively. Zoom in the pictures to see the difference.

\subsection{Geometry motion flow}

Let the time-varying surfaces $P=\left(P_{1}, P_{2}, \ldots, P_{T}\right)$ be a sequence of $T$ frame surfaces, where $P_{t} \in R^{3}$ is the $t$ th frame surface with $n_{t}$ points, we refer to the index $t$ as "time". Let $F$ be a feature on $P$. In our method, the feature $F$ is a connected subpart of the surface $P_{t}$, and is usually a non-trivial local shape and contains salient parts of the model. The boundaries of the feature are usually edges of the model. Inspired by the optic flow computation in image sequences $[35,36,37,38]$, we track the geometry feature $F$ by propagating the initial frame $F_{0}$ of $F$ along the motion flow field $\vec{V}$ of $P$ to the next successive frames.

To estimate flow field between frame $P_{t}$ and its successive frame $P_{t+\delta t}$ for propagating the geometry feature $F$, we consider a point $p(x, y, z, t)$ in $P_{t}$ with normal $N(x, y, z, t)$, where $(x, y, z)$ denotes the location of $p$, and $t \in[0, T]$ denotes time (frame). Let $N(x+\delta x, y+\delta y, z+\delta z, t+\delta t)$ denote the normal of its corresponding point $p(x+\delta x, y+\delta y, z+\delta z, t+\delta t)$ in frame $P_{t+\delta t}$. The motion flow computation requires both the position of the points and their normals. More specially, the motion flow computation for each point $p$ on $P$ is based on normal constancy assumption, that is, we assume the 
deviation $\delta$ from normal constancy is minimal for each point of the feature $F$ in the subsequent frames:

$$
\delta=N(x+\delta x, y+\delta y, z+\delta z, t+\delta t)-N(x, y, z, t) .
$$

The deviation $\delta$ can be approximated by using the first order Taylor expansion: $\delta=N_{x} u+N_{y} v+N_{z} w+N_{t}$, where $N_{x}, N_{y}, N_{z}$ and $N_{t}$ are vectors of $3 \times 1$, and denote the normal gradient of $N$ in $x, y, z, t$ axis, respectively. More specially, $N_{x}$ is computed as the difference between the normal of $p$ and the average normal of the spatially neighboring points of point $p$ in the $X$ axis direction, and $N_{y}$ and $N_{z}$ are defined in the same way. $N_{t}$ is computed as the difference of normal of $p$ and the average normal of the points in the temporal neighborhood of $p$. The three dimensional vector $\vec{v}_{p}=(u, v, w)$ are the 3D motion flow vector of point $p$, where $u=\delta x / \delta t, v=\delta y / \delta t$, $w=\delta z / \delta t$. Assume that $\delta=(0,0,0)$, we have $N_{x} u+N_{y} v+N_{z} w=-N_{t}$, which is considered as motion flow constraint for motion flow computation. Motion flow $\vec{v}_{p}$ computed in this strategy is called local flow computation method.

However, the local method does not allow a reliable determination of the full motion flow, especially at the homogeneous regions on the timevarying surfaces, which may result in nondense flow fields. Inspired by Horn and Schunck's global optical flow computation in image sequences [35], the neighboring points should have similar motion velocities in regions where the surface varies smoothly almost everywhere. We express this as to minimize the square of the magnitude of the gradient of the motion flow vector $\vec{v}$, and add an additional smoothing regulation term to the local method. We come to minimize the following energy function:

$$
\begin{aligned}
& F=\int_{D}\left(N_{x} u+N_{y} v+N_{z} w+N_{t}\right)^{2} \\
& +\alpha^{2}\left(|\nabla u|^{2}+|\nabla v|^{2}+|\nabla w|^{2}\right) d x d y d z,
\end{aligned}
$$

where $D$ is the spatio-temporal neighborhood domain of point $p$ over the surfaces $P_{t}$, and the magnitude of $\alpha$ reflects the relative influence of the regulation term. $\nabla u=\left(u_{x}, u_{y}, u_{z}\right)$ are the $3 \mathrm{D}$ velocity derivatives at point $p(x, y, z, t), u_{x}$ is computed as the difference between $u$ and the average $u$ value of the spatial neighboring points of point $p$ in the $X$ axis direction, similar definition for $u_{y}$ and $u_{z}$. $\nabla v$ and $\nabla w$ can be defined in the same way.

Minimizing the functional Equ.(1) can be obtained by solving its corresponding Euler-Lagrange equations. Let $N_{x} N_{y}$ be the dot product between 
two vectors: $N_{x} \cdot N_{y}$, and $N_{x}^{2}$ be dot product between vectors: $N_{x} \cdot N_{x}$, similar definition for other vectors, we can rewrite the Euler-Lagrange equations as:

$$
\begin{aligned}
& N_{x}^{2} u+N_{x} N_{y} v+N_{x} N_{z} w+N_{x} N_{t}=\alpha^{2} \nabla^{2} u \\
& N_{x} N_{y} u+N_{y}^{2} v+N_{y} N_{z} w+N_{y} N_{t}=\alpha^{2} \nabla^{2} v \\
& N_{x} N_{z} u+N_{y} N_{z} v+N_{z}^{2} w+N_{z} N_{t}=\alpha^{2} \nabla^{2} w .
\end{aligned}
$$

By approximating $\nabla^{2} u \approx \bar{u}-u, \nabla^{2} v \approx \bar{v}-v, \nabla^{2} w \approx \bar{w}-w$, where $\bar{u}, \bar{v}$ and $\bar{w}$ are three components of the $\overline{\vec{v}}$, and $\overline{\vec{v}}$ is the velocity average of those points in the spatial neighborhood of point $p$ in current frame. Then we receive:

$$
\begin{aligned}
\left(\alpha^{2}+N_{x}^{2}\right) u+N_{x} N_{y} v+N_{x} N_{z} w & =\alpha^{2} \bar{u}-N_{x} N_{t} \\
N_{x} N_{y} u+\left(\alpha^{2}+N_{y}^{2}\right) v+N_{y} N_{z} w & =\alpha^{2} \bar{v}-N_{y} N_{t} \\
N_{x} N_{z} u+N_{y} N_{z} v+\left(\alpha^{2}+N_{z}^{2}\right) w & =\alpha^{2} \bar{w}-N_{z} N_{t} .
\end{aligned}
$$

This can be rewritten in an alternative form:

$$
\begin{aligned}
\left(\alpha^{2}+N_{x}^{2}+N_{y}^{2}+N_{z}^{2}\right)(u-\bar{u}) & =-N_{x}\left(N_{x} \bar{u}+N_{y} \bar{v}+N_{z} \bar{w}+N_{t}\right) \\
\left(\alpha^{2}+N_{x}^{2}+N_{y}^{2}+N_{z}^{2}\right)(v-\bar{v}) & =-N_{y}\left(N_{x} \bar{u}+N_{y} \bar{v}+N_{z} \bar{w}+N_{t}\right) \\
\left(\alpha^{2}+N_{x}^{2}+N_{y}^{2}+N_{z}^{2}\right)(w-\bar{w}) & =-N_{z}\left(N_{x} \bar{u}+N_{y} \bar{v}+N_{z} \bar{w}+N_{t}\right) .
\end{aligned}
$$

We can use Gauss-Seidel iteration to solve motion flow field $\vec{v}_{p}$ from above equations, which can be performed in the following formulation:

$$
\begin{aligned}
u^{k+1} & =\bar{u}^{k}-\frac{N_{x}\left(N_{x} \bar{u}^{k}+N_{y} \bar{v}^{k}+N_{z} \bar{w}^{k}+N_{t}\right)}{\alpha^{2}+N_{x}^{2}+N_{y}^{2}+N_{z}^{2}} \\
v^{k+1} & =\bar{v}^{k}-\frac{N_{y}\left(N_{x} \bar{u}^{k}+N_{y} \bar{v}^{k}+N_{z} \bar{w}^{k}+N_{t}\right)}{\alpha^{2}+N_{x}^{2}+N_{y}^{2}+N_{z}^{2}} \\
w^{k+1} & =\bar{w}^{k}-\frac{N_{z}\left(N_{x} \bar{u}^{k}+N_{y} \bar{v}^{k}+N_{z} \bar{w}^{k}+N_{t}\right)}{\alpha^{2}+N_{x}^{2}+N_{y}^{2}+N_{z}^{2}} .
\end{aligned}
$$

The $\bar{u}^{k}, \bar{v}^{k}$ and $\bar{w}^{k}$ are velocity averages of points in the neighborhoods at iteration $k$. The initial values of $\bar{u}^{0}, \bar{v}^{0}$ and $\bar{w}^{0}$ are typically set to 0.0 . The motion flow field $\vec{v}_{p}$ also can be solved using multi-grid method, which can be accelerated about 50 times than Gauss-Seidel method. 


\subsection{Multi-scale geometry motion flow}

The geometry details and noises of time-varying surfaces will perturb the geometry motion flow computation. To make the motion flow computation more robust and receive more accurate results, we build the motion flow models over the coarser time-varying surfaces with the detail layers attenuated. Since progressively coarser levels increases the degree of smoothness in the resulting time-varying surfaces, we build motion flow for each level of timevarying surfaces and combine them together in a spatially varying manner to provide more consistent motion flow results. Based on this observation, we present a multi-scale optical flow method.

Inspired by geometry bilateral filter [39], we first compute multi-scale representation for the time-varying surfaces. For the input time-varying surface $P$, the goal of the multi-scale spatio-temporal bilateral filtering is to build a series of filtered surfaces $P^{j}$ that preserve the strongest features while smoothing small changes in geometry. At the finest scale $j=0$, we set $P^{0}=P$ and then iteratively apply the bilateral filter to compute

$$
p^{j+1}=\frac{1}{k_{p^{j}}} \sum_{q^{j} \in D\left(p^{j}\right)} G_{\sigma_{s}}\left(\left\|p^{j}-q^{j}\right\|\right) \cdot G_{\sigma_{r}}\left(\left\langle n_{p}^{j}, p^{j}-q^{j}\right\rangle\right) \cdot q^{j}
$$

with

$$
k_{p^{j}}=\sum_{q^{j} \in D\left(p^{j}\right)} G_{\sigma_{s}}\left(\left\|p^{j}-q^{j}\right\|\right) \cdot G_{\sigma_{r}}\left(\left\langle n_{p}^{j}, p^{j}-q^{j}\right\rangle\right),
$$

where $p^{j}$ and $q^{j}$ denote the points $p$ and $q$ at scale $j . D(p)$ is the spatiotemporal neighborhood of vertex $p$ and $G_{\sigma}$ is Gaussians function, and $\sigma_{s}$ and $\sigma_{r}$ are the widths of the spatial and range Gaussians respectively. In our experiments, $\sigma_{s}$ is set to the radius of the spatio-temporal neighborhood, and $\sigma_{r}$ is set to the standard deviation of the offsets $\left\langle n_{p}^{j}, p^{j}-q^{j}\right\rangle$ in the neighborhood. In our experiments, the size of the spatio-temporal neighborhood includes 12-18 points, which depends on the sampling density of the point set. For dense point set, the size is set relative large value, and for coarse point set, the size is set relative small value. If the data set is filtered using weighted average of large size of points, the features of the point set may be smoothed. With the filtered time-varying surfaces, we then compute motion flows $\vec{v}^{j}$ for each scale representation $P^{j}$ of the time-varying surfaces.

To make the $j$ th scale motion flow $\vec{v}^{j}$ smoother, we propose a motion flow vector filtering function that incorporating the information from both spatial 
and temporal domain in each scale of time-varying surface. Let $D^{*}(p)$ be the spatio-temporal neighborhood of point $p, D_{t}^{*}(p) \in D^{*}(p)$ be the neighborhood of point $p$ from the $t$ th frame, $k$ be the number of the neighboring frames of $t$ th frame in time axis $\left(k=2\right.$ in our experiments). For each scale $P^{j}$ of time-varying surfaces, we compute the optimized motion flow $\vec{v}_{p}^{j}$ for each surface frame as follows:

$$
\vec{v}_{p}^{j}=\frac{\sum_{f=t-k}^{t+k} \sum_{q \in D_{f}^{*}(p)} G_{\sigma_{s}}(\|p-q\|) \bullet G_{\sigma_{r}}\left(\left\|N_{p}-N_{q}\right\|\right) \bullet \vec{v}_{q}^{j}}{\sum_{f=t-k}^{t+k} \sum_{q \in D_{f}^{*}(p)} G_{\sigma_{s}}(\|p-q\|) \bullet G_{\sigma_{r}}\left(\left\|N_{p}-N_{q}\right\|\right)} .
$$

With the computed optical flows $\vec{v}^{j}$ for each point in each scale, we compute the final geometry flow of the point $p$ as the weighted average of motion flow in each scale:

$$
\vec{v}_{p}=\frac{\sum_{j=0}^{M-1} \omega_{j} \vec{v}_{p}^{j}}{\sum_{i=0}^{M-1} \omega_{j}}
$$

where $\omega_{j}$ is weight for each scale and is defined as $\omega_{j}=1.2^{j}-0.6$, and $M$ is the number of the scales. The weight $\omega_{j}$ prefers coarser surface by giving them larger weight because the geometry noise are smoothed more. In our experiments, we find that by setting number $M=4$ generates good results.

In Fig.1 and Fig.2, we give the comparison results between the motion flow computed on the single-scale strategy and multi-scale strategy. As illustrated in Fig.1 and Fig.2, the motion flow computed on multi-scale time-varying surfaces are smoother and more accurate.

After computing the motion flow $\vec{v}_{p}=(u, v, w)$ for each point $p(x, y, z, t)$ on frame $P_{t}$, we can find its corresponding point in the next frame $P_{t+1}$ by propagating this point along its motion flow $\vec{v}_{p}$. For point $p$, we compute its propagated points along $\vec{v}_{p}$ as $p^{\prime}=p+\vec{v}_{p}$, then we search a point $p^{*}$ in $P_{t+1}$ that are nearest to point $p^{\prime}$. We apply the ANN method [40] for nearest neighboring point search, and the $\epsilon$ used in ANN is chosen as 0.3. Using this method, for each frame $F_{t}$ in feature $F$, we can find its corresponding feature frame $F_{t+1}$ in $P_{t+1}$, and the feature $F$ propagates along the motion flow.

Note that using this feature propagation method, there are such case that some points in feature frame $F_{t+1}$ can not find corresponding points in $F_{t}$, for 

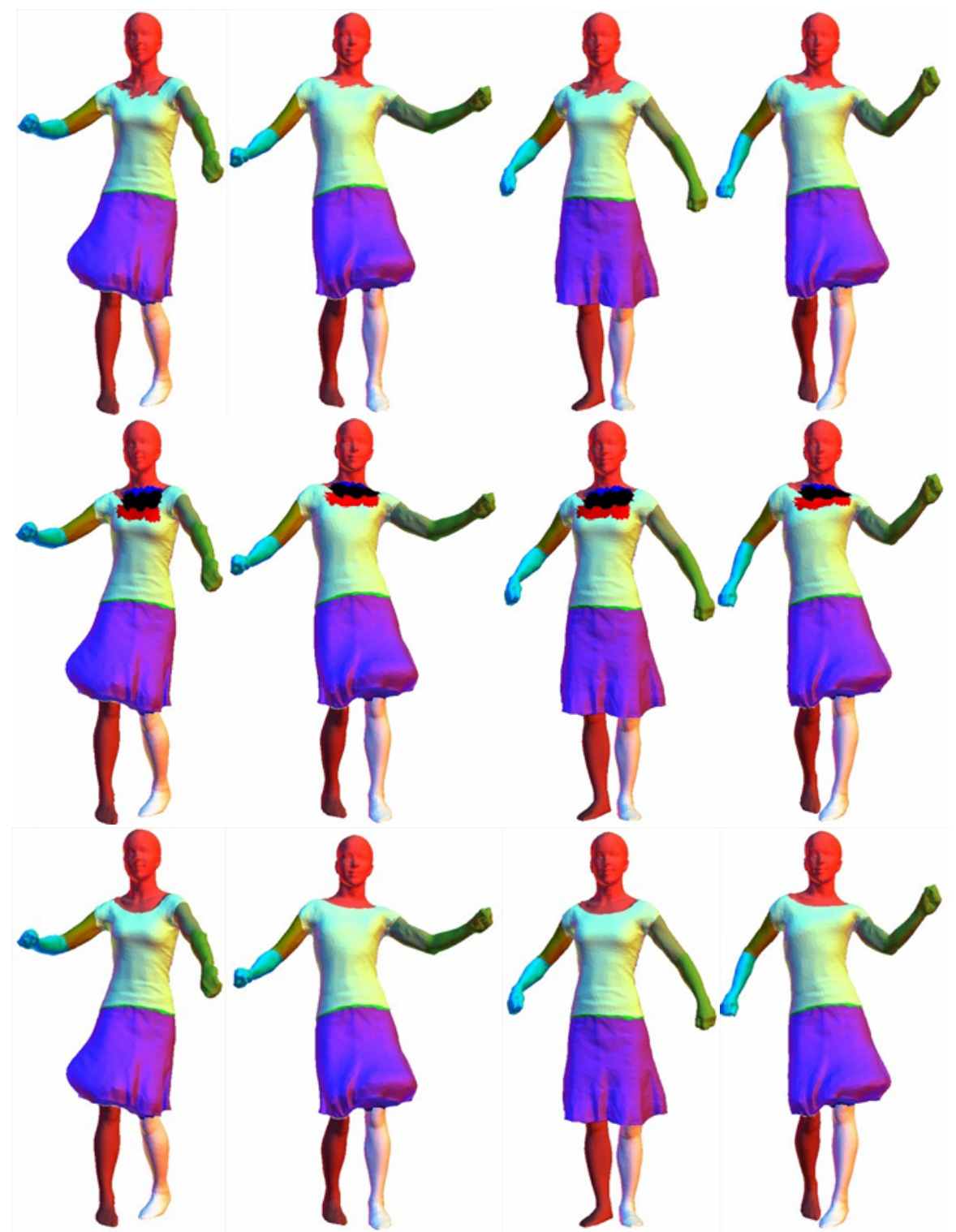

Figure 3: Boundaries refinement. The 1st row: the initial feature boundaries, the 2st row: the boundary band, foreground band and background band built on the models, the 3st row: the boundaries refinement results.

example, when the features are becoming larger. In this case, we apply the local flood filling techniques to make $F_{t+1}$ coherent and compact. For each 
point $p$ in $F_{t+1}$ that have corresponding point in $F_{t}$, we detect its nearboring points in $F_{t+1}$ that have no corresponding points in $F_{t}$, and include these points in the feature.

\subsection{Feature boundaries refinement}

The dynamic features can be tracked effectively using above tracking method, however, sometimes the feature boundaries may look rough and not smooth, which need to be further refined.

Inspired by [41], Lee et al. [2] applied the maximum flow minimum cut algorithm to process boundaries refinement of deforming mesh. However, this method is applicable for triangle mesh, and is inappropriate for pointsampled geometry. Furthermore, it process the boundaries smoothing frame by frame, thus it is difficult to maintain the boundaries temporally coherent. In this section, inspired by [4], we apply a probability optimization method to generate convincing and coherent results.

The main idea of our boundaries smoothing operations is to use the geometric information (normal) and motion velocities around the initial boundaries to determine the accurate boundaries [4]. As shown in Fig. 3, a narrow boundary band $W$ is built around the initial boundaries which envelops the whole boundaries. Our target is to decide the accurate boundaries in $W$. The size of $W$ can vary with regard to the size of the object features.

We build both foreground band $F$ and background band $B$ regions around $W$, and build a Gaussian Mixture Models (GMMs) for both $F$ and $B$ using the geometric information and motion velocities. Every point $p$ inside the band $W$ can be assigned a foreground probability. Depending on the probability value belonging to the foreground $F$ or the background $B$, the accurate boundaries can be determined.

For a point $p$ in the band $W$, its foreground probability $G M M_{F}(p)$ is computed as follows: $\operatorname{GMM}_{F}(p)=G M M(p \mid F) /(G M M(p \mid F)+G M M(p \mid B))$ where $G M M(p \mid F)$ and $G M M(p \mid B)$ are the corresponding probabilities for point $p$ computed from the two GMMs for $F$ and $B$, respectively. The background probability $G M M_{B}(p)$ of point $p$ is defined in similar way. Then, the point $p$ can be classified efficiently by evaluating foreground and background probability value: if $G M M_{B}(p)>G M M_{F}(p)$, point $p$ belongs to the foreground $F$, otherwise $p$ belongs to background $B$. Note to keep the boundaries of the dynamic feature temporally coherent, both foreground band $F$ and background band $B$ should be spatio-temporal neighborhood of current frame. More details refer to [4] and [42]. 
The size of $W$ can vary according to the complexity of the boundary. Usually, in our experiments, for each point on the boundary, its nearest 10 points are included in $W$, and receive satisfactory results. As illustrated in Fig.3, using the proposed boundaries refinement operator, the initial boundaries of dynamic features are refined to receive more accurate results.

\subsection{Feature propagation using surfaces matching}

For time-varying surfaces in large deformation with low frame sampling rate, we also integrate shape matching into our system for obtaining more accurate feature tracking results. To perform surfaces matching, for two successive frames $P_{t}$ and $P_{t+1}$, we first estimate SIFT feature points [24] for each frame of the time-varying surfaces, then incorporating both the SIFT feature points and the motion flow vectors, we perform the multi-scale surface matching.

More specially, inspired by the SIFT method [23, 24], we extract salien$\mathrm{t}$ feature points on the time-varying surfaces for surface matching. These feature points need not to represent visually important portions of the timevarying surfaces, but be a stable set of feature points for surface matching. As illustrated in Fig.4, we detect the salient features for each frame of the time-varying surfaces. We can observe that the most salient features exist on each frame. These feature points have several attributes (the position, the normal, and the scale) that can be used to perform surface matching. However, these attributes are not invariant under shape rigid transformation or scaling. In order to enable efficient matching for the surfaces, we endow each feature point $p$ with a signature vector $\sigma_{p}(p, n, h)$ that is invariant under shape rigid transformation or scaling [24]. By applying the repeatability of the feature points on surfaces, we compare the feature sets with signature set for shape matching.

To perform surface matching between the neighboring frames, for each frame $P_{t}$, based on the detected surface feature points and their corresponding signature vectors, we compute a transformation combined rotation matrix $R_{t}$ and translation vector $S_{t}$. With this transformation we matches $P_{t}$ with its consequent frame $P_{t+1}$, such that $P_{t}$ overlap $P_{t+1}$ within some common regions. For more accurate matching, we can combine both the neighboring frame information of $P_{t-1}$ and $P_{t+2}$ into surface matching. With the surface matching results, for each point in the current frame, we can find more accurate spatio-temporal neighborhood used in Equ. 1 for motion flow estimation 


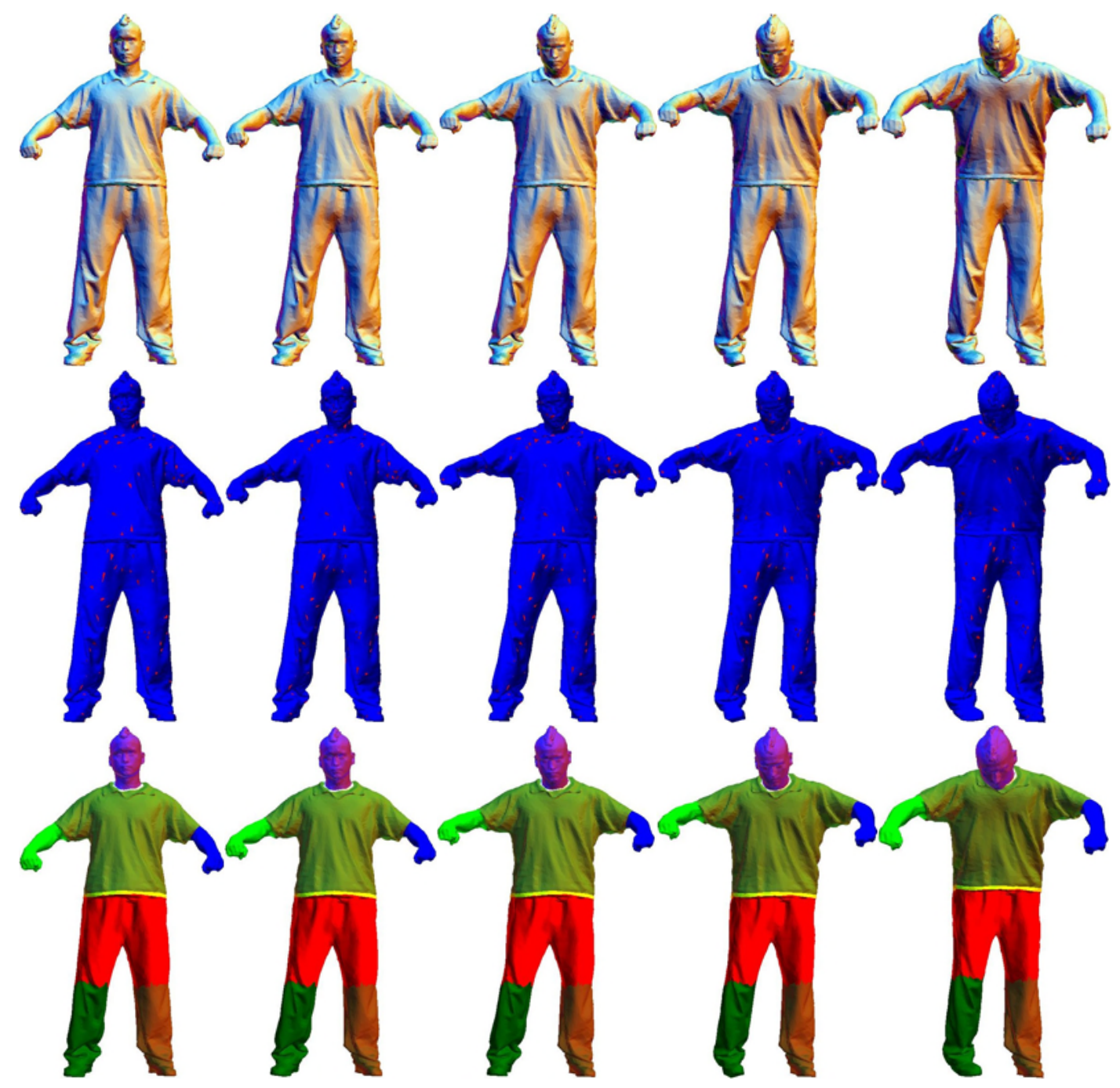

Figure 4: Feature propagation using surfaces matching. Top row, 5 successive frames from the input time-varying surfaces. Middle row, detected SIFT features (red points) on the surface frames. Bottom row, features tracking results incorporating surfaces matching.

from $P_{t}$ to $P_{t+1}$, which is more accurate than the results computing without using surfaces matching.

As illustrated in Fig.4, we present a feature tracking example for a timevarying surfaces in large deformation with low frame sampling rate, the features are effectively tracked out. 


\subsection{Multi-frame propagation}

So far we have only described a forward propagation workflow, where the user sets an initial starting surface for the dynamic feature, and lets the features propagate forward. The features tracking generated by the single-frame propagation system may contain errors. These errors might lead to more errors in the next frame if a simple frame-by-frame propagation is adopted, especially for processing time-varying surfaces with large deformation, fuzzy boundary region, features splitting and merging, or time-varying surfaces with long sequence. Thus, if no constraints are applied, the tracked dynamic features might gradually drift away, resulting in amplified errors in the propagation process. For example, dynamic objects often present topology changes, a robust system needs to be able to handle these topological changes. Inspired by video object tracking [9], in this section, we present multi-scale propagation to address these problems.

Alternatively, we can define two key frames for the dynamic feature $F$ at time $P_{s}$ and $P_{t}$ (we assume $P_{s}$ comes before $P_{t}$ in the time order), and track all intermediate frames using both constraints. To achieve this we carry out two individual propagation processes: a forward propagation from $P_{s}$ to $P_{t}$, and a backward propagation from $P_{t}$ to $P_{s}$. For an intermediate frame $r$, the two processes merge. Formally, the feature tracking map on frame $r$ is computed as $p_{F}^{r}(\vec{v})=\frac{(r-s) \cdot p_{F}^{s \rightarrow r}(\vec{v})+(t-r) \cdot p_{F}^{t \rightarrow r}(\vec{v})}{t-s}$, where $p_{F}^{s \rightarrow r}(\vec{v})$ and $p_{F}^{t \rightarrow r}(\vec{v})$ are the corresponding feature tracking maps for frame $r$ computed from the forward and backward processes, respectively. The tracking map $p_{F}^{r}(\vec{v})$ is more accurate than $p_{F}^{s \rightarrow r}(\vec{v})$ and $p_{F}^{t \rightarrow r}(\vec{v})$, which results in more accurate tracking results. For more complex time-varying surfaces, we can specify more keyframes on the feature. With the single-frame propagation system and multi-frame propagation system, our system thus has the flexibility of switching between different workflows based on the user's choice.

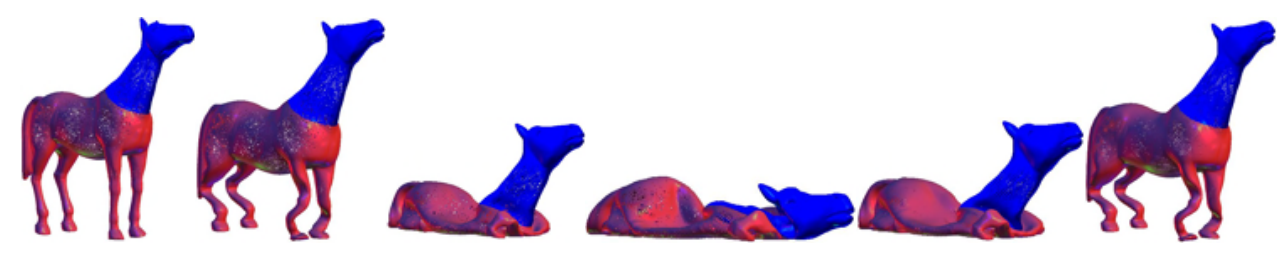

Figure 5: Feature tracking results using multi-frame propagation system.

Fig.5 shows feature tracking results on time-varying surfaces consisting 
of 110 frames. In this example, we track a disappearing and reappearing feature using the presented multi-frame propagation system, and achieve desired results.

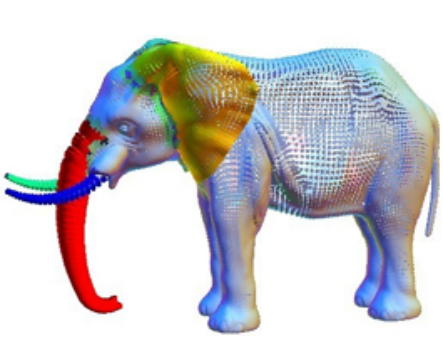

(a)

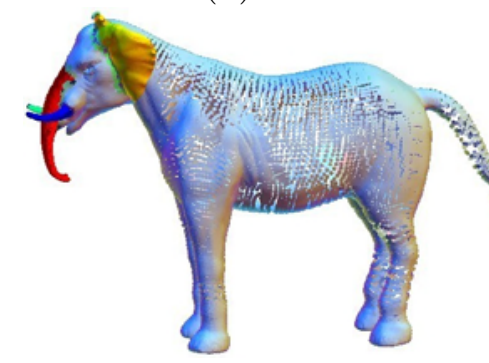

(d)

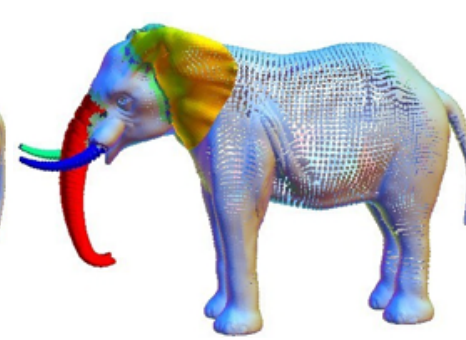

(b)

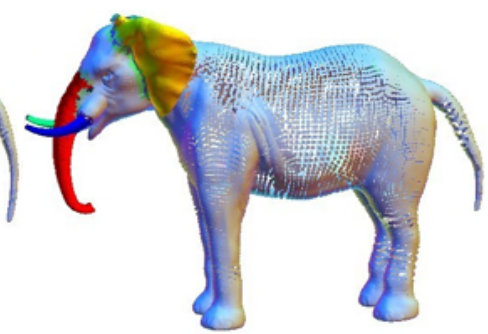

(c)

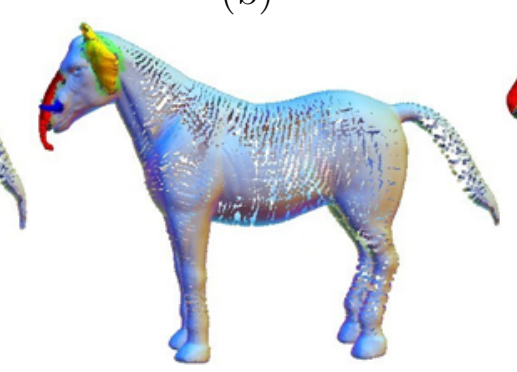

(e)

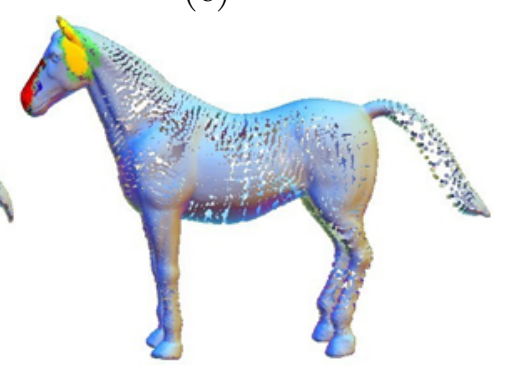

(f)

Figure 6: Multi object features tracking for a morphing sequence from elephant to horse. (a) Initial frame, (b)-(f)tracking results of three features.

\section{Experimental results and discussion}

In this section, we present experimental results received using the presented method, and provide feature tracking examples for both mesh based and point based time-varying surfaces. We also compare our algorithm to related works on both performance and quality. Our approach is implemented using $\mathrm{C}++$ on a machine equipped with Pentium (R) Dual-Core CPU E5200@2.50GHz with 2GB RAM.

In Fig.5, we present a tracking result using the multi-frame propagation method for a mesh-based time-varying surfaces with 110 frames. As the specified feature is disappearing and reappearing, single-frame propagation does not work well for this case, we apply the multi-frame propagation method to 
track the feature. In this example, we use the first and the last frame as the key frame, and receive satisfactory results.

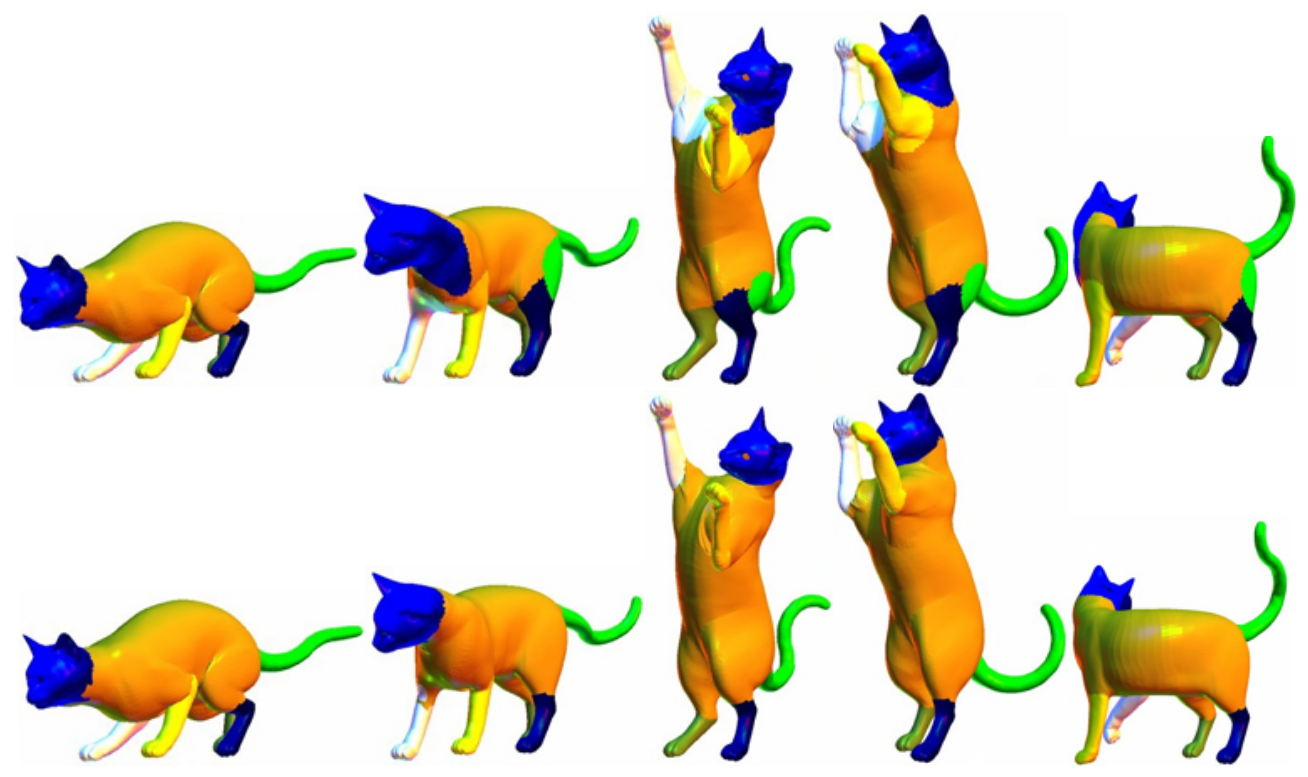

Figure 7: Feature tracking results comparison. Top row: feature tracking results of [15], bottom row, feature tracking results using our multi-frame propagation method.

In Fig.6, we give multi-features tracking results for point based timevarying surfaces, which are elephant to horse morphing sequences. We track three object features, tusk, trunk and ears of elephant. The time-varying surfaces consist of 201 frame surfaces. The number of sampled points in each frame is not identical, and the averaged number of points in each frame is 85,000 . The computational time for each frame in the tracking process is 1.89 seconds. In this example, we use the simple single-frame propagation and do not employ the multi-frame propagation method, we also do not apply shape matching in the feature tracking procedure.

In Fig. 7 and Fig.8, we give comparison results with the feature tracking results of method [15]. Cagniart et al. [15] considered deformation of the time-varying surfaces using patches and assumed that each one transformed rigidly. In addition, Cagniart et al. [15] needed a reference mesh to constrain the topological nature of the time-varying surfaces for feature tracking. In these examples, the features have large deformation and some feature are rigid. As shown in Fig.7 and Fig.8, our method can achieve the better feature 


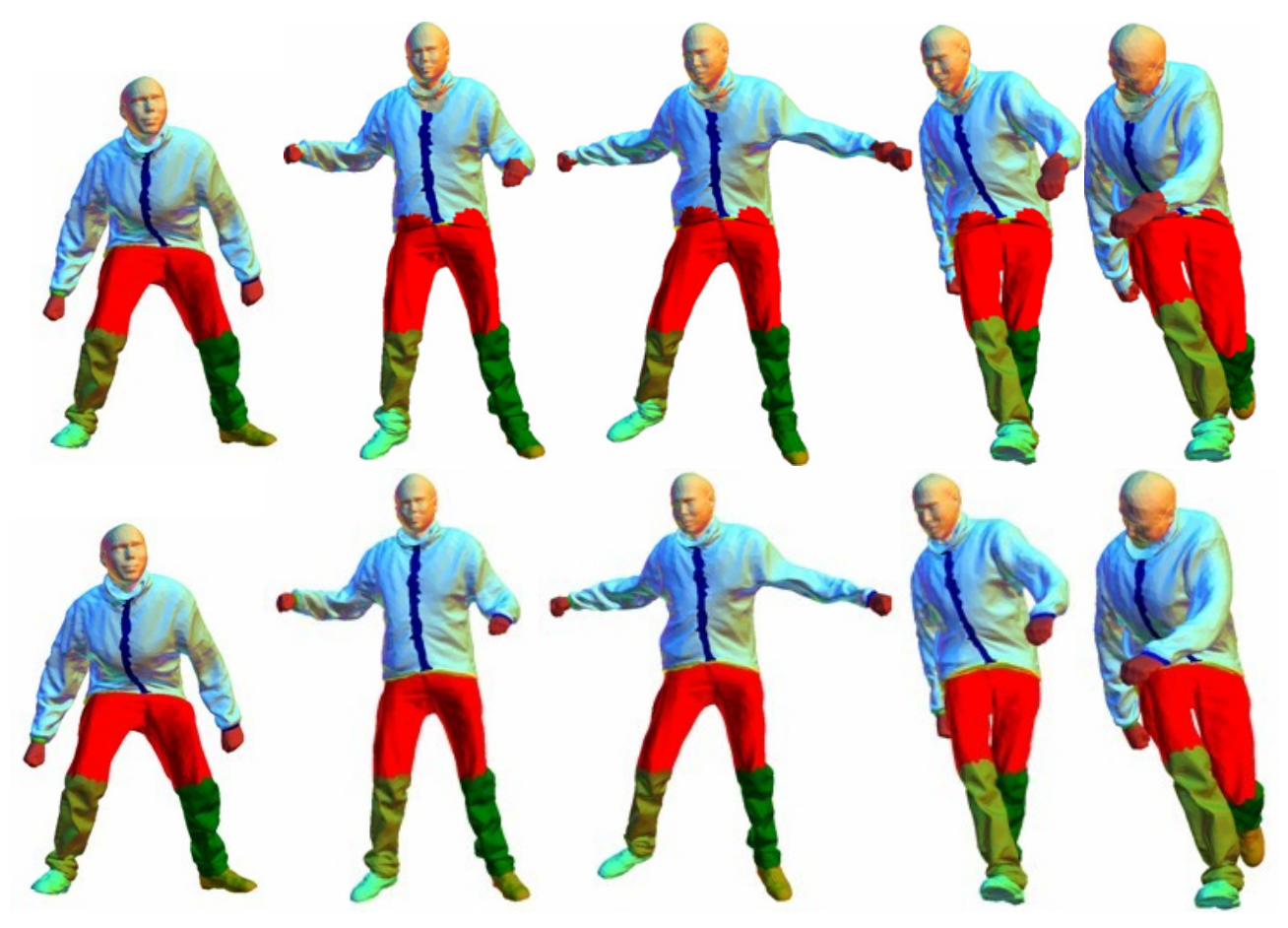

Figure 8: Feature tracking comparison. Top row: feature tracking results of [15], bottom row, feature tracking results using our multi-frame propagation method.

tracking results than [15]. In Fig.7 and Fig.8, the time-varying cat surfaces consist of $30 \backslash 80$ frame surfaces, the averaged number of vertices in each frame is $17,800 \backslash 42,300$, and the cost time using our method for tracking each frame is $0.62 \backslash 0.92$ seconds, respectively.

In Fig.9, we give a feature tracking result for point-based time-varying face surfaces. We track three object features, left eye, right eye and mouth. The time-varying surfaces consist of 385 frame surfaces. In this example, the boundaries of the features are fuzzy, and the surface sequence is long, the errors occurs in one frame might lead to more errors in the next frame if a single-frame propagation is adopted. We use multi-frame propagation and select three key frames. We give comparison results with the segmentation results of the time-varying surfaces [4]. Liao et al.[4] considered the timevarying surfaces as a whole and extracted the near-rigid components. In these example, the features are non-rigid components, although method [4] works well in time-varying surfaces segmentation, it is not a ideal tool for 


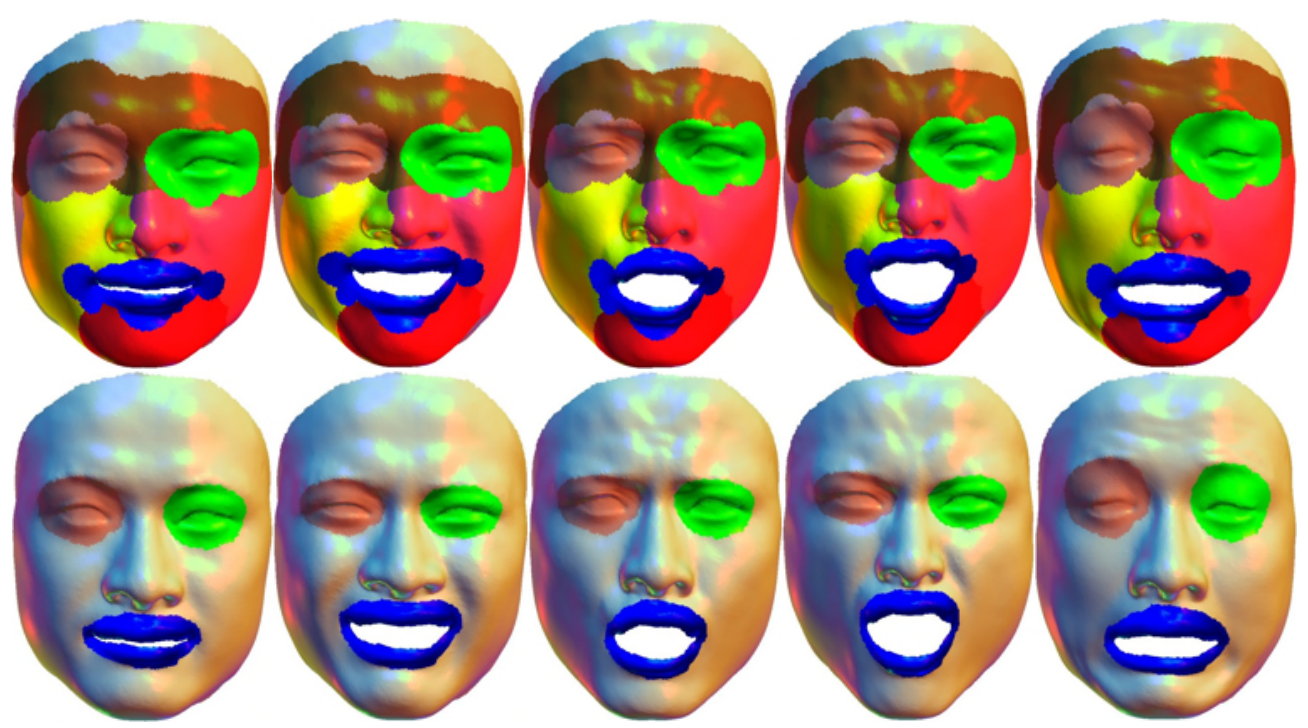

Figure 9: Feature tracking result comparison with segmentation result of [4] for timevarying face surfaces. Top row: segmentation result of [4]. Bottom row: feature tracking result using our method (The features are specified manually in the key frames).

tracking non-rigid features. The averaged number of vertices in each frame is 23,725 , the cost time using our method for tracking each frame is 0.73 seconds.

In Fig.10, we present feature tracking results for another point-based based time-varying surfaces with non-rigid components. In this example, we track three features on the deforming penguin. The feature tacking methods that focus on tracking the rigid components may not work well on this example. Our feature tracking method based on geometry motion flow is appropriate tool for this example. As illustrated in Fig.10, the three features are effectively tracked out.

Fig.11 and Fig.12 give more feature tracking results for two dancing timevarying surfaces. In these two examples, we first perform segmentation process for the first (key) frame using the interactive mesh segmentation method [33], and segment it into several parts and each part is considered a feature. As the time-varying surfaces are in large deformation with low frame sampling rate, we integrate the SIFT features matching into our system for obtaining effective feature tracking results. In Fig.11 and Fig.12, there are 90 and 101 frames in the animations, and the average 31,775, 35,648 points 


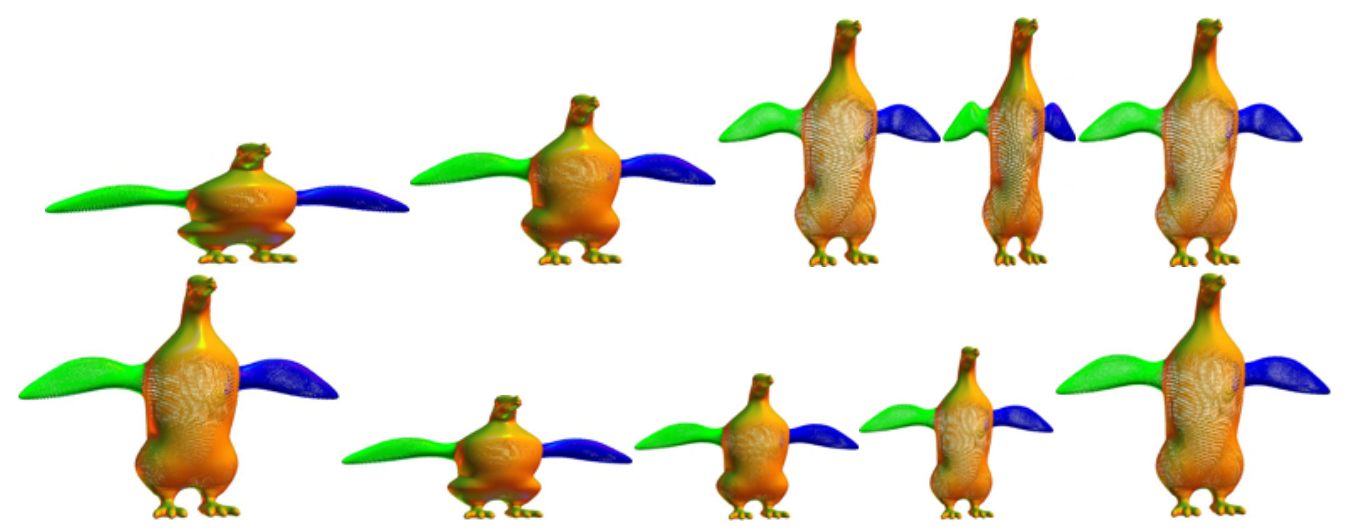

Figure 10: Non-rigid components tracking on time-varying surfaces.

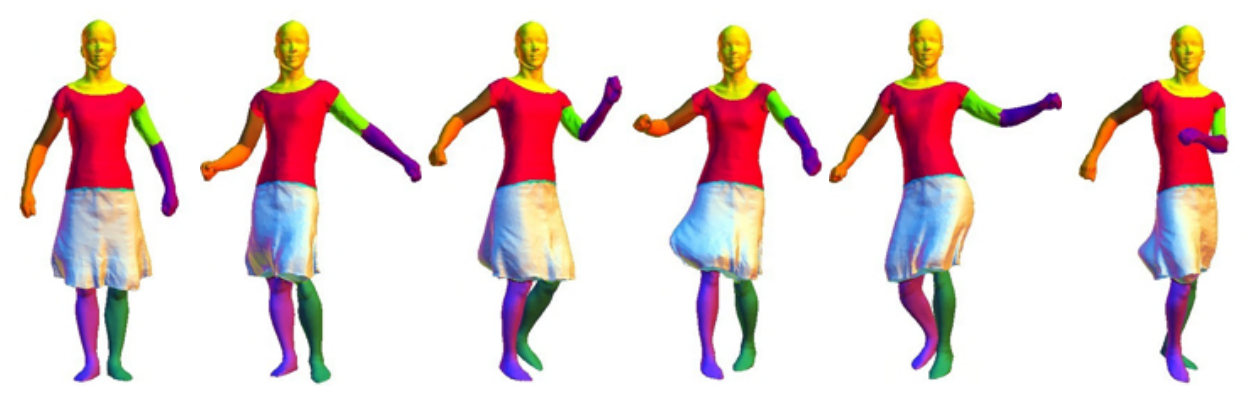

Figure 11: Multi-features tracking incorporating shape matching.

in each frame, respectively. We detect about 321 and 343 feature points for each frame for shape matching in these two examples, respectively. As the input time-varying surfaces are smooth, we use two-scale representation for computing the motion-flow and find that works well. We also apply the GM$\mathrm{M}$ boundaries refinement to compute accurate boundaries, and takes about 0.78 and 0.83 seconds to perform the boundaries processing.

In Fig.13 and Fig.14, we have performed feature tracking processing on the data with highly varying numbers of vertices. The data is from INRIA (http : // 4drepository.inrialpes.fr/public/datasets). In these examples, the original surfaces from INRIA are triangular meshes. Note that in these models, the motions of individual vertex are not smooth, local geometry features almost change in a short time window. To show the robustness of the proposed method, we further resample the input data into two kinds of 


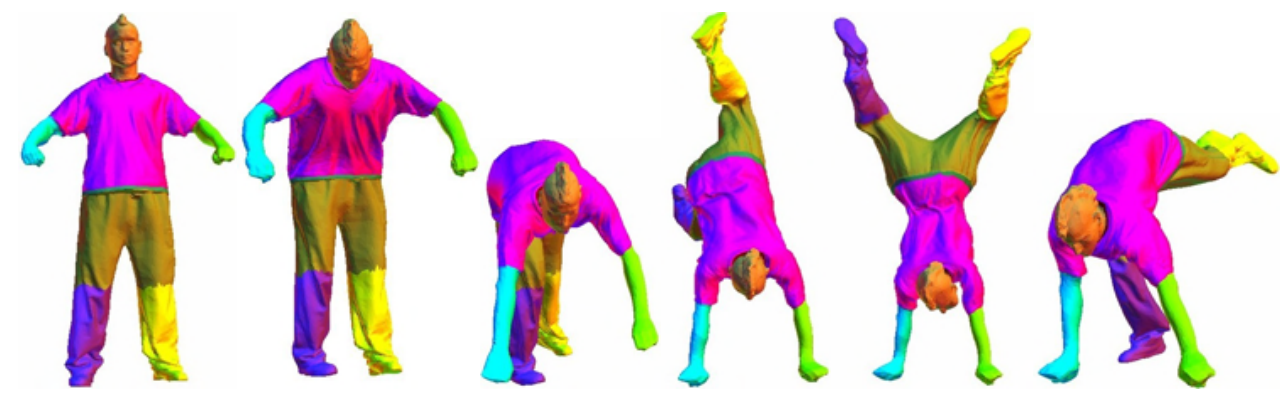

Figure 12: Multi-features tracking incorporating shape matching.

point set, and one kind of resampling result is uniform sampling, and one kind of resampling result is non-uniform sampling. In Fig.13 and Fig.14, the minimum points of one surface frame are 5,704,5,336, respectively, and maximum points are 59,853, 82,914, respectively. In Fig.13, we tracked the moving head and the two moving arms for both kinds of data, and received pleasing results for these two kinds of data. In Fig.14, we track the leg of a moving character. In these examples, as the time-varying surfaces are in large deformation with different frame sampling rate, we integrate both shape matching and multi-frame propagation into our system for computing more accurate feature tracking results.

In Fig.15 and Fig.16, we give feature tracking results on the time-varying surfaces captured by the Microsoft Kinect. The data set captured by the Kinect usually contains noise, and there is no temporal coherence (vertex to vertex correspondence) between the frames. Furthermore, the input data set is not moving smooth, as shown in the supplementary video, the original captured time-varying surfaces are flickering. In Fig.15, we give the tracking results on both depth image sequence (point-based time-varying surfaces) and the results for the depth images with corresponding textures (RGB images). In this example, we tracking the motion of the arm and receive satisfactory results. In Fig.16, we track the one feature of the deforming face, and when the face is deforming, the moving feature is effectively tracked.

Table 1 presents the average, minimum, and maximum number of the vertices for each frame of time-varying surfaces.

Limitations: When decomposing the animated objects with extremely large deformation while with small amounts of surface sampling frames, as shown the collapsing camel in Fig.17, our method can not work very well. In 


\begin{tabular}{|l|l|l|l|}
\hline Model & Average number & Minimum number & Maximum number \\
\hline Fig.6 & 85,000 & 32,789 & 100,693 \\
Fig.7 & 17,800 & 16,998 & 18,668 \\
Fig.8 & 42,300 & 40,032 & 44,374 \\
Fig.9 & 23,725 & 22,239 & 24,113 \\
Fig.10 & 33,635 & 32,457 & 33,992 \\
Fig.11 & 31,775 & 30,664 & 32,489 \\
Fig.12 & 35,648 & 34,117 & 36,887 \\
Fig.13 & 32,247 & 5,704 & 59,853 \\
Fig.14 & 45,137 & 5,336 & 82,914 \\
Fig.15 & 55,182 & 34,183 & 62,298 \\
Fig.16 & 31,221 & 25,234 & 45,346 \\
\hline
\end{tabular}

Table 1: The average, minimum, and maximum number of the vertices for each frame of time-varying surfaces.

this example, we track 6 components of the camel, including its 4 legs. As in this case, the shape deformation in each frame is large, even the adjacent frames are matched in high accuracy, and the overlapped regions between adjacent frames is small (limited), for example, the leg regions. Thus, the points in the time-varying surfaces can not find desirable neighboring points for motion flow computation, which results in inaccurate motion flow computation, especially on the regions with large deformation.

\section{Conclusion and future work}

In this paper, we have presented a framework for efficient feature tracking of time-varying surfaces. We define an effective multi-scale geometry motion flow for the time-varying surfaces, which efficiently propagates the geometry features on the time-varying surfaces. By combining both the approximately invariant signature vectors and geometry motion flow vectors, we also corporate shape matching techniques into our system to process feature tracking for time-varying surfaces in large deformation with low frame sampling rate. Furthermore, multi-frame propagation system also has been developed to improve our feature tracking results.

In the future, we will report the following progress in latter publications. Firstly, optical flow computation has been widely investigated by computer 


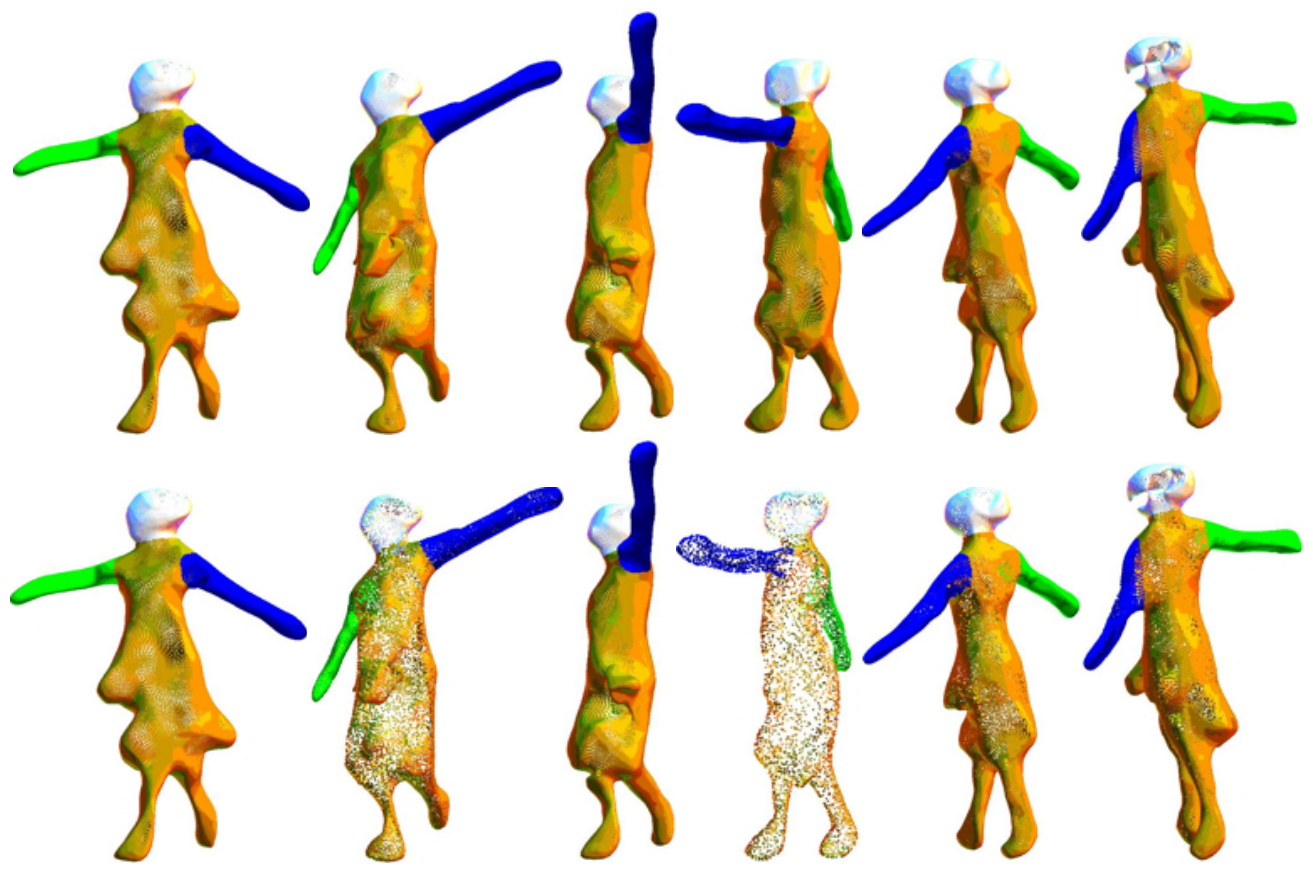

Figure 13: Feature tracking on the time-varying surfaces. Top row, feature tracking result on uniformly sampled data set. Bottom row, feature tracking result on non-uniformly sampled data set.

vision researchers. When the objects in the input image sequences have the occlusion artifacts, these methods cannot correctly handle the flow estimation for the occluded region. Similarly, due to the occlusion and low surface albedo, scanned surface sequences usually exhibit large holes, such as the scanned data from Microsoft Kinect. In this case, motion flow computing for geometric features may not be accurate due to geometry incompletion in time-varying surfaces. We would like to develop a more stable motion flow computation to address this issue. Secondly, though SIFT matching of object features work well, the matching efficiency needs to be addressed when data amount of time-varying surfaces is much large.

\section{Acknowledgment}

We would like to thank the reviewers for their comments, we also thank Robert W. Sumner, Li Zhang and many other researchers for presenting the 


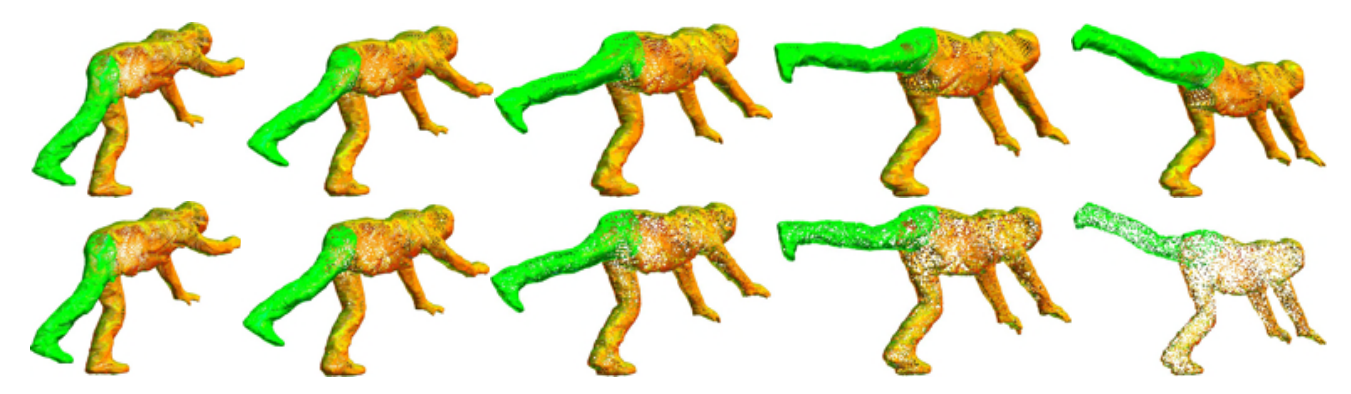

Figure 14: Feature tracking on the time-varying surfaces. Top row, feature tracking result on uniformly sampled data set. Bottom row, feature tracking result on non-uniformly sampled data set.

mesh and point cloud data.

\section{References}

[1] Li H, Luo L, Vlasic D, Peers P, Popović J, Pauly M, et al. Temporally coherent completion of dynamic shapes. ACM Transactions on Graphics (TOG) 2012;31(1):2.

[2] Lee T, Wang Y, Chen T. Segmenting a deforming mesh into near-rigid components. The Visual Computer 2006;22(9):729-39.

[3] Wuhrer S, Brunton A. Segmenting animated objects into near-rigid components. The Visual Computer 2010;26(2):147-55.

[4] Liao B, Xiao C, Liu M, Dong Z, Peng Q. Fast hierarchical animated object decomposition using approximately invariant signature. The Visual Computer 2012;28(4):387-99.

[5] Mitra N, Flory S, Ovsjanikov M, Gelfand N, Guibas L, Pottmann H. Dynamic geometry registration. In: ACM International Conference Proceeding Series; vol. 257. 2007, p. 173-82.

[6] Wand M, Jenke P, Huang Q, Bokeloh M, Guibas L, Schilling A. Reconstruction of deforming geometry from time-varying point clouds. In: ACM International Conference Proceeding Series; vol. 257. Citeseer; 2007, p. 49-58. 


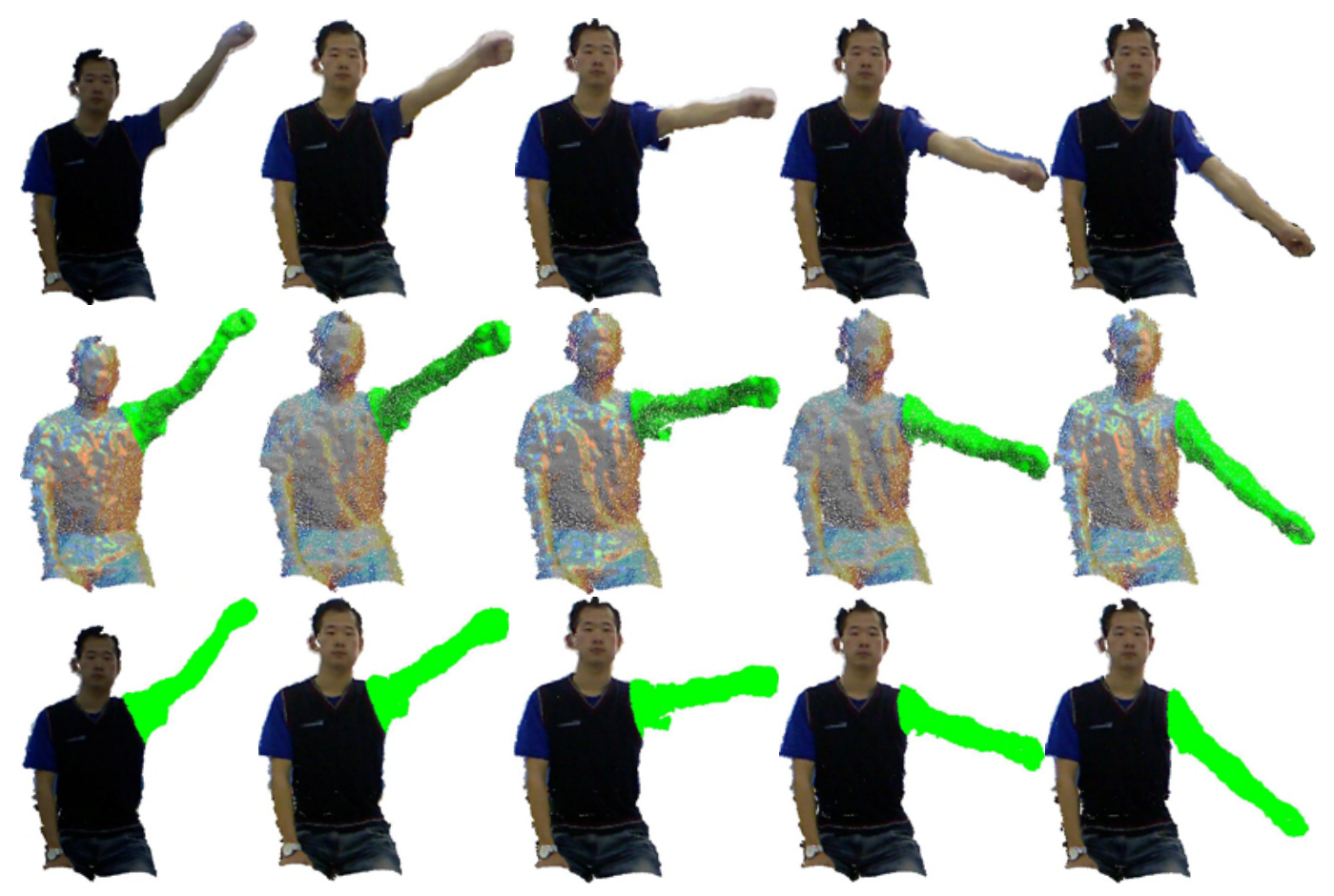

Figure 15: Feature tracking on the time-varying surfaces captured by Microsoft Kinect. Top row, input data with both time-varying depth images (point cloud) and corresponding RGB textures. Middle row, tracking result for the time-varying depth images. Bottom row, attaching the RGB textures on the tracked depth images.

[7] Kircher S, Garland M. Editing arbitrarily deforming surface animations. In: ACM Transactions on Graphics (TOG); vol. 25. ACM; 2006, p. 1098-107.

[8] Xu W, Zhou K, Yu Y, Tan Q, Peng Q, Guo B. Gradient domain editing of deforming mesh sequences. In: ACM Transactions on Graphics (TOG); vol. 26. ACM; 2007, p. 84.

[9] Bai X, Wang J, Simons D, Sapiro G. Video snapcut: robust video object cutout using localized classifiers. In: ACM Transactions on Graphics (TOG); vol. 28. ACM; 2009, p. 70.

[10] Vlasic D, Baran I, Matusik W, Popović J. Articulated mesh animation 


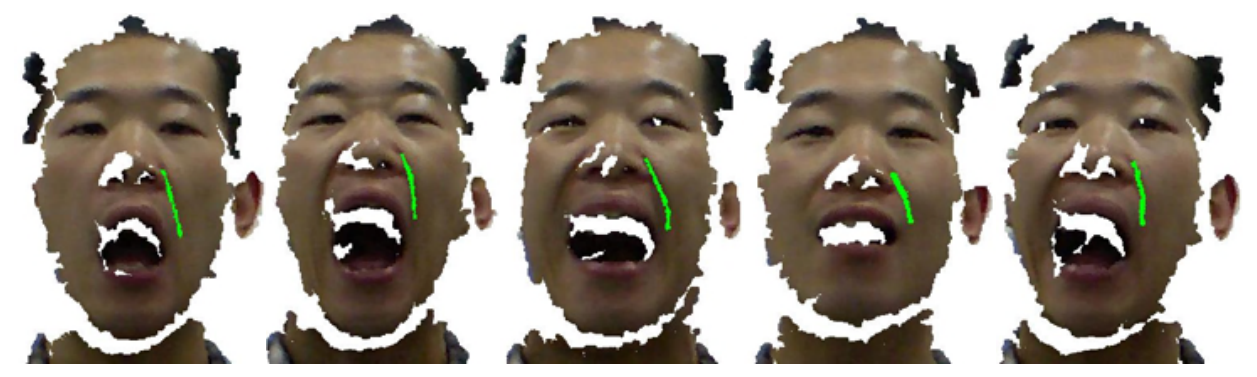

Figure 16: Feature tracking on the time-varying surfaces captured by Microsoft Kinect.

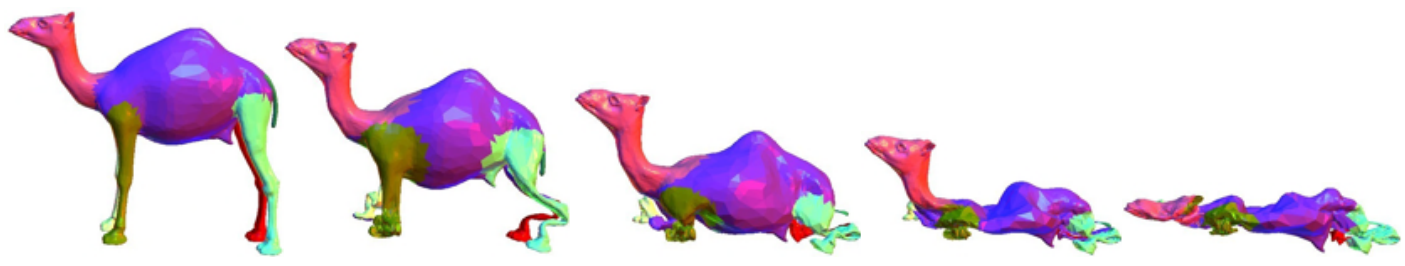

Figure 17: Feature tracking for time-varying surfaces in large deformation.

from multi-view silhouettes. In: ACM Transactions on Graphics (TOG); vol. 27. ACM; 2008, p. 97.

[11] Gall J, Stoll C, De Aguiar E, Theobalt C, Rosenhahn B, Seidel H. Motion capture using joint skeleton tracking and surface estimation. In: Computer Vision and Pattern Recognition, 2009. CVPR 2009. IEEE Conference on. IEEE; 2009, p. 1746-53.

[12] Chai J, Xiao J, Hodgins J. Vision-based control of 3d facial animation. In: Proceedings of the 2003 ACM SIGGRAPH/Eurographics symposium on Computer animation. Eurographics Association; 2003, p. 193206.

[13] Salzmann M, Pilet J, Ilic S, Fua P. Surface deformation models for nonrigid 3d shape recovery. Pattern Analysis and Machine Intelligence, IEEE Transactions on 2007;29(8):1481-7.

[14] Grinspun E, Hirani A, Desbrun M, Schröder P. Discrete shells. In: Proceedings of the 2003 ACM SIGGRAPH/Eurographics symposium on Computer animation. Eurographics Association; 2003, p. 62-7. 
[15] Cagniart C, Boyer E, Ilic S. Free-form mesh tracking: a patch-based approach. In: Computer Vision and Pattern Recognition (CVPR), 2010 IEEE Conference on. IEEE; 2010, p. 1339-46.

[16] Osher S, Fedkiw R. Level set methods and dynamic implicit surfaces; vol. 153. Springer; 2002.

[17] Enright D, Marschner S, Fedkiw R. Animation and rendering of complex water surfaces. ACM Transactions on Graphics (TOG) 2002;21(3):73644 .

[18] Wojtan C, Thürey N, Gross M, Turk G. Deforming meshes that split and merge. In: ACM Transactions on Graphics (TOG); vol. 28. ACM; 2009 , p. 76.

[19] Bojsen-Hansen M, Li H, Wojtan C. Tracking surfaces with evolving topology. ACM Transactions on Graphics (TOG) 2012;31(4):53.

[20] Shapira L, Shalom S, Shamir A, Cohen-Or D, Zhang H. Contextual part analogies in $3 \mathrm{~d}$ objects. International Journal of Computer Vision 2010;89(2):309-26.

[21] De Aguiar E, Theobalt C, Stoll C, Seidel H. Marker-less 3d feature tracking for mesh-based human motion capture. In 2nd Workshop on Human Motion-Understanding, Modeling, Capture and Animation 2007;:1-15.

[22] Franco J, Boyer E. Learning temporally consistent rigidities. In: Computer Vision and Pattern Recognition (CVPR), 2011 IEEE Conference on. IEEE; 2011, p. 1241-8.

[23] Lowe D. Distinctive image features from scale-invariant keypoints. International journal of computer vision 2004;60(2):91-110.

[24] Li X, Guskov I. Multi-scale features for approximate alignment of pointbased surfaces. In: Proceedings of the third Eurographics symposium on Geometry processing; vol. 217. Citeseer; 2005,

[25] Wand M, Adams B, Ovsjanikov M, Berner A, Bokeloh M, Jenke P, et al. Efficient reconstruction of nonrigid shape and motion from real-time $3 \mathrm{~d}$ scanner data. ACM Transactions on Graphics (TOG) 2009;28(2):15. 
[26] Torresani L, Yang D, Alexander E, Bregler C. Tracking and modeling non-rigid objects with rank constraints. In: Computer Vision and Pattern Recognition, 2001. CVPR 2001.Proceedings of the 2001 IEEE Computer Society Conference on; vol. 1. IEEE; 2001, p. 493-.

[27] Sharf A, Alcantara D, Lewiner T, Greif C, Sheffer A, Amenta N, et al. Space-time surface reconstruction using incompressible flow. In: ACM Transactions on Graphics (TOG); vol. 27. ACM; 2008, p. 110.

[28] Bradley D, Popa T, Sheffer A, Heidrich W, Boubekeur T. Markerless garment capture. In: ACM Transactions on Graphics (TOG); vol. 27. ACM; 2008, p. 99.

[29] De Aguiar E, Stoll C, Theobalt C, Ahmed N, Seidel H, Thrun S. Performance capture from sparse multi-view video. In: ACM Transactions on Graphics (TOG); vol. 27. ACM; 2008, p. 98.

[30] Bradley D, Heidrich W, Popa T, Sheffer A. High resolution passive facial performance capture. ACM Transactions on Graphics (TOG) 2010;29(4):41.

[31] Beeler T, Hahn F, Bradley D, Bickel B, Beardsley P, Gotsman C, et al. High-quality passive facial performance capture using anchor frames. In: ACM Transactions on Graphics (TOG); vol. 30. ACM; 2011, p. 75.

[32] Valgaerts L, Wu C, Bruhn A, Seidel H, Theobalt C. Lightweight binocular facial performance capture under uncontrolled lighting. ACM Transactions on Graphics (TOG) 2012;31(6):187.

[33] Xiao C, Fu H, Tai C. Hierarchical aggregation for efficient shape extraction. The Visual Computer 2009;25(3):267-78.

[34] Fan L, Meng M, Liu L. Sketch-based mesh cutting: A comparative study. Graphical Models 2012;

[35] Horn B, Schunck B. Determining optical flow. Artificial intelligence 1981;17(1):185-203.

[36] Bruhn A, Weickert J, Schnörr C. Combining the advantages of local and global optic flow methods. Pattern Recognition 2002;:454-62. 
[37] Lucas B, Kanade T, et al. An iterative image registration technique with an application to stereo vision. In: Proceedings of the 7th international joint conference on Artificial intelligence. 1981,.

[38] Xiao J, Cheng H, Sawhney H, Rao C, Isnardi M. Bilateral filtering-based optical flow estimation with occlusion detection. Computer VisionECCV 2006 2006;:211-24.

[39] Fleishman S, Drori I, Cohen-Or D. Bilateral mesh denoising. In: ACM Transactions on Graphics (TOG); vol. 22. ACM; 2003, p. 950-3.

[40] Arya S, Mount D, Netanyahu N, Silverman R, Wu A. An optimal algorithm for approximate nearest neighbor searching fixed dimensions. Journal of the ACM (JACM) 1998;45(6):891-923.

[41] Katz S, Leifman G, Tal A. Mesh segmentation using feature point and core extraction. The Visual Computer 2005;21(8):649-58.

[42] Xiao C, Nie Y, Tang F. Efficient edit propagation using hierarchical data structure. Visualization and Computer Graphics, IEEE Transactions on 2011;17(8):1135-47.

\section{Appendix}

We give more technical details for geometry motion flow computation.

Minimising the functional (1) comes down to solving its corresponding Euler-Lagrange equations. They are given by:

$$
\begin{gathered}
F_{u}-\frac{d}{d x} F_{u_{x}}-\frac{d}{d y} F_{u_{y}}-\frac{d}{d z} F_{u_{z}}-\frac{d}{d t} F_{u_{t}}=0 \\
F_{v}-\frac{d}{d x} F_{v_{x}}-\frac{d}{d y} F_{v_{y}}-\frac{d}{d z} F_{v_{z}}-\frac{d}{d t} F_{v_{t}}=0 \\
F_{w}-\frac{d}{d x} F_{w_{x}}-\frac{d}{d y} F_{w_{y}}-\frac{d}{d z} F_{w_{z}}-\frac{d}{d t} F_{w_{t}}=0
\end{gathered}
$$

where:

$$
\begin{aligned}
& F_{u}=2 N_{x}\left(N_{x} u+N_{y} v+N_{z} w+N_{t}\right), \\
& F_{v}=2 N_{y}\left(N_{x} u+N_{y} v+N_{z} w+N_{t}\right),
\end{aligned}
$$




$$
\begin{gathered}
F_{w}=2 N_{z}\left(N_{x} u+N_{y} v+N_{z} w+N_{t}\right), \\
F_{u_{x}}=2 \alpha^{2} u_{x}, F_{u_{y}}=2 \alpha^{2} u_{y}, F_{u_{z}}=2 \alpha^{2} u_{z}, F_{u_{t}}=2 \alpha^{2} u_{t}, \\
F_{v_{x}}=2 \alpha^{2} v_{x}, F_{v_{y}}=2 \alpha^{2} v_{y}, F_{v_{z}}=2 \alpha^{2} v_{z}, F_{v_{t}}=2 \alpha^{2} v_{t}, \\
F_{w_{x}}=2 \alpha^{2} w_{x}, F_{w_{y}}=2 \alpha^{2} w_{y}, F_{w_{z}}=2 \alpha^{2} w_{z}, F_{w_{t}}=2 \alpha^{2} w_{t},
\end{gathered}
$$

and

$$
\begin{gathered}
\frac{d F_{u_{x}}}{d x}=2 \alpha^{2} u_{x x}, \frac{d F_{u_{y}}}{d y}=2 \alpha^{2} u_{y y}, \frac{d F_{u_{z}}}{d z}=2 \alpha^{2} u_{z z}, \frac{d F_{u_{t}}}{d t}=2 \alpha^{2} u_{t t}, \\
\frac{d F_{v_{x}}}{d x}=2 \alpha^{2} v_{x x}, \frac{d F_{v_{y}}}{d y}=2 \alpha^{2} v_{y y}, \frac{d F_{v_{z}}}{d z}=2 \alpha^{2} v_{z z}, \frac{d F_{v_{t}}}{d t}=2 \alpha^{2} v_{t t} \\
\frac{d F_{w_{x}}}{d x}=2 \alpha^{2} w_{x x}, \frac{d F_{w_{y}}}{d y}=2 \alpha^{2} w_{y y}, \frac{d F_{w_{z}}}{d z}=2 \alpha^{2} w_{z z}, \frac{d F_{w_{t}}}{d z}=2 \alpha^{2} w_{t t} .
\end{gathered}
$$

Since $\nabla^{2} u=u_{x x}+u_{y y}+u_{z z}+u_{t t}, \nabla^{2} v=v_{x x}+v_{y y}+v_{z z}+v_{t t}, \nabla^{2} w=$ $w_{x x}+w_{y y}+w_{z z}+w_{t t}$, we can rewrite the Euler-Lagrange equations as:

$$
\begin{aligned}
& N_{x}^{2} u+N_{x} N_{y} v+N_{x} N_{z} w+N_{x} N_{t}=\alpha^{2} \nabla^{2} u \\
& N_{x} N_{y} u+N_{y}^{2} w+N_{y} N_{z} w+N_{y} N_{t}=\alpha^{2} \nabla^{2} v \\
& N_{x} N_{z} u+N_{y} N_{z} v+N_{z}^{2} w+N_{z} N_{t}=\alpha^{2} \nabla^{2} w .
\end{aligned}
$$

Using $\nabla^{2} u \approx \bar{u}-u, \nabla^{2} v \approx \bar{v}-v, \nabla^{2} w \approx \bar{w}-w$, where $\bar{u}, \bar{v}$ and $\bar{w}$ are the averages of neighborhoods of velocities, we can receive:

$$
\begin{aligned}
& \left(\alpha^{2}+N_{x}^{2}\right) u+N_{x} N_{y} v+N_{x} N_{z} w=\alpha^{2} \bar{u}-N_{x} N_{t} \\
& N_{x} N_{y} u+\left(\alpha^{2}+N_{y}^{2}\right) v+N_{y} N_{z} w=\alpha^{2} \bar{v}-N_{y} N_{t} \\
& N_{x} N_{z} u+N_{y} N_{z} v+\left(\alpha^{2}+N_{z}^{2}\right) w=\alpha^{2} \bar{w}-N_{z} N_{t} .
\end{aligned}
$$

We can solve for $u, v$ and $w$ as:

$$
\begin{aligned}
\left(\alpha^{2}+N_{x}^{2}+N_{y}^{2}+N_{z}^{2}\right) u & =\left(\alpha^{2}+N_{y}^{2}+N_{z}^{2}\right) \bar{u}-N_{x} N_{y} \bar{v}-N_{x} N_{z} \bar{w}-N_{x} N_{t} \\
\left(\alpha^{2}+N_{x}^{2}+N_{y}^{2}+N_{z}^{2}\right) v & =\left(\alpha^{2}+N_{x}^{2}+N_{z}^{2}\right) \bar{v}-N_{x} N_{y} \bar{u}-N_{y} N_{z} \bar{w}-N_{y} N_{t} \\
\left(\alpha^{2}+N_{x}^{2}+N_{y}^{2}+N_{z}^{2}\right) w & =\left(\alpha^{2}+N_{x}^{2}+N_{y}^{2}\right) \bar{w}-N_{x} N_{z} \bar{u}-N_{y} N_{z} \bar{v}-N_{z} N_{t},
\end{aligned}
$$


The above equations can be written as:

$$
\begin{aligned}
\left(\alpha^{2}+N_{x}^{2}+N_{y}^{2}+N_{z}^{2}\right)(u-\bar{u}) & =-N_{x}\left(N_{x} \bar{u}+N_{y} \bar{v}+N_{z} \bar{w}+N_{t}\right) \\
\left(\alpha^{2}+N_{x}^{2}+N_{y}^{2}+N_{z}^{2}\right)(v-\bar{v}) & =-N_{y}\left(N_{x} \bar{u}+N_{y} \bar{v}+N_{z} \bar{w}+N_{t}\right) \\
\left(\alpha^{2}+N_{x}^{2}+N_{y}^{2}+N_{z}^{2}\right)(w-\bar{w}) & =-N_{z}\left(N_{x} \bar{u}+N_{y} \bar{v}+N_{z} \bar{w}+N_{t}\right) .
\end{aligned}
$$

We can use the Gauss-Seidel iteration method to solve above equations:

$$
\begin{aligned}
u^{k+1} & =\bar{u}^{k}-\frac{N_{x}\left(N_{x} \bar{u}^{k}+N_{y} \bar{v}^{k}+N_{z} \bar{w}^{k}+N_{t}\right)}{\alpha^{2}+N_{x}^{2}+N_{y}^{2}+N_{z}^{2}} \\
v^{k+1} & =\bar{v}^{k}-\frac{N_{y}\left(N_{x} \bar{u}^{k}+N_{y} \bar{v}^{k}+N_{z} \bar{w}^{k}+N_{t}\right)}{\alpha^{2}+N_{x}^{2}+N_{y}^{2}+N_{z}^{2}} \\
w^{k+1} & =\bar{w}^{k}-\frac{N_{z}\left(N_{x} \bar{u}^{k}+N_{y} \bar{v}^{k}+N_{z} \bar{w}^{k}+N_{t}\right)}{\alpha^{2}+N_{x}^{2}+N_{y}^{2}+N_{z}^{2}} .
\end{aligned}
$$

$\bar{u}^{k}, \bar{v}^{k}$ and $\bar{w}^{k}$ are averages of neighborhoods of velocities at iteration $k$, and typically set to 0.0 . 